\title{
Electron Transfer and Solvent Mediated Electronic Localization in Molecular Photocatalysis
}

\author{
Asmus O. Dohn, ${ }^{, \dagger}$ Kasper S. Kjær, ${ }^{\ddagger}$ Tobias B. Harlang, Sophie E. Canton, \\ Martin M. Nielsen,,$\ddagger$ and Klaus B. Møller ${ }^{\dagger}$ \\ $\dagger$ Department of Chemistry, Technical University of Denmark, Kemitorvet 206, 2800 Kgs \\ Lyngby, Denmark \\ $\ddagger$ Department of Physics, Technical University of Denmark, Building 3072800 Kgs. \\ Lyngby, Denmark \\ IDeutsches Elektronensynchrotron DESY, Notkestrasse 85, D-22607 Hamburg, Germany \\ E-mail: asod@kemi.dtu.dk
}

\begin{abstract}
This work provides a detailed mechanism for electron transfer in a hetero-dinuclear complex designed as a model system in which to study homogeneous molecular photocatalysis. With efficient Born-Oppenheimer Molecular Dynamics simulations, we show how intermediate, charge separated states can mediate the electron transfer. We observe how Jahn-Teller distortion effects play out in solution, when the molecule has energetically close-lying states and how this distortion is averaged out in the thermal sampling. Finally, we demonstrate how the solvent helps stabilize and localize the separated charge. The information on the electronic configuration and separate states is of key importance for designing next generation photocatalysts.
\end{abstract}




\section{Introduction}

The development of robust systems for harnessing solar energy as carbon-neutral solar fuels is a key challenge for modern society. Molecular systems are attractive candidates for developing solar fuels production due to the inherent selectivity and tunability of molecular photocatalytic reactions. However, the attractive features of molecular photocatalysis couples to significant complexity in reaction mechanisms. Thus, in order to take advantage of the tunability and selectivity of molecular systems to rationally improve existing or design new photocatalytic molecules, detailed knowledge of existing systems and the mechanisms within them are required. Recent years have seen a proliferation of ultrafast experimental methods providing unprecedented insight into light-induced molecular processes. ${ }^{1-8}$ The presented conclusions on underlying mechanisms and their origins are typically being arrived at by correlating experimental observations with predictions of computational chemistry. The computational framework of choice is often a minimisation-based approach to molecular modelling, where an atomistic description is solely applied to the molecule of interest, providing a static representation of minimum energies and nuclear structures. ${ }^{9-15}$ The level of theory, ranging from DFT to more purely ab initio post-Hartree Fock or multi-reference methods, is then chosen to balance computational cost with model-intrinsic accuracy for the problem at hand. However, whichever level of theory is chosen, the static energy minimization-type of molecular model does not directly provide thermally averaged structures, cannot reliably describe cases where more than one electronic configuration is thermally accessible, and cannot provide insights into specific solute/solvent interactions. One way of addressing these shortcomings is by interfacing quantum mechanical calculations with explicit solvent molecules described within a molecular mechanics framework (QM/MM calculations) and propagating

the system under ambient conditions, using molecular dynamics (MD). ${ }^{16-22}$ However, simulations within this type of framework are very costly, since the electronic density has to be fully re-converged for every classical time step (Born-Oppenheimer MD, BOMD), or the time steps have to be very small (Carr-Parrinello MD), either way resulting in a reduction 


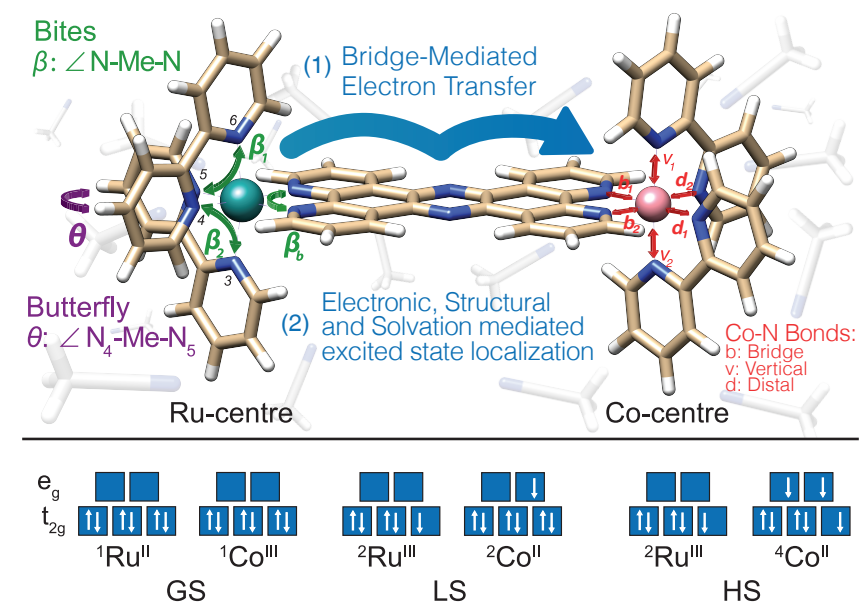

Figure 1: The relevant bond definitions of $\left[(\mathrm{bpy})_{2} \mathrm{Ru}(\mathrm{tpphz}) \mathrm{Co}(\mathrm{bpy})_{2}\right]^{5+}$, or $[\mathrm{Ru}=\mathrm{Co}]$, for brevity, and the electronic configurations examined.

in the generality of the findings from such frameworks. We have recently addressed this by developing a fast implementation of a DFT-based, QM/MM BOMD method ${ }^{23-25}$. Utilizing the efficiency of a real-space implementation of the Projector Augmented Wave (PAW) method, ${ }^{26-28}$ it is possible to rapidly and robustly model photoactive complexes comprised of up to hundreds of atoms, their environments, and to make it computationally viable to produce multiple-trajectory statistics for more reliable comparisons to experimental data. Solvent and solvation dynamics have been shown to be a key factor in describing charge transfer between, and in the stabilization of excited states. ${ }^{29,30}$ Especially when the solute changes size during the ET process, translational solvent molecule motions have been shown to be of key importance, making it necessary to go beyond the dielectric continuum solvent approximation. ${ }^{29}$

In this work, we describe the BOMD exploration of charge transfer and solvation of a large hetero-dinuclear complex designed to act as a model system for homogeneous molecular photocatalysis. These systems contain, within one molecule, three principle components: A photoabsorber, a catalytic center and bridging unit that enables electron transfer between absorber and catalyst. ${ }^{31}$ In recent years, many reports have been made on such model systems, and similar complexes. ${ }^{31-40}$ The system under investigation $\left[(\text { bpy })_{2} \mathrm{Ru}^{\mathrm{II}}(\mathrm{tpphz}) \mathrm{Co}^{\mathrm{III}}(\mathrm{bpy})_{2}\right]^{5+}{ }_{-}$ 
(where bpy $=2,2$ - bipyridine, tpphz $=$ tetrapyrido $(3,2$ - a:2'3'-c:3",2" - h::2"',3"' - j) phenazine (abbreviated as "[Ru=Co]") is shown in figure 1.

$[\mathrm{Ru}=\mathrm{Co}]$ represents one of the most thoroughly investigated system within the broad class of photoactive Ruthenium/Cobalt heterodinuclear molecular systems designed towards photocatalysis. Recent studies have shown that selective excitation of the Ruthenium center leads to ultra-fast charge transfer to the Cobalt site in a long-lived $\left[{ }^{2} \mathrm{Ru}^{\mathrm{III}}={ }^{4} \mathrm{Co}^{\mathrm{II}}\right]$ state: Experiments suggest that the charge transfer cascade involves a phenazine-bridge localized state, and an intermediate ${ }^{2} \mathrm{Co}{ }^{\mathrm{II}}$ state preceding the formation of the final ${ }^{4} \mathrm{Co}^{\mathrm{II}}$ charge separated state. ${ }^{3,41}$ Here, the involvement of the intermediate is confirmed, and we furthermore address the underlying mechanisms for the ultra-fast, efficient photoinduced charge transfer, and long-lived charge separation exhibited by this complex. We conduct a thorough investigation of the properties of the $\left[{ }^{2} \mathrm{Ru}^{\mathrm{III}}={ }^{2} \mathrm{Co}^{\mathrm{II}}\right]$ intermediate state, which is centered around a Co-center that is optically dark, and too short lived for exhaustive direct experimental characterization using x-ray methods. The investigation identifies it as a key component in the ultrafast charge transfer cascade. We show how the nature of the solvation changes with the charge transfer, but also highlight that the two charge separated states $\left(\left[{ }^{2} \mathrm{Ru}^{\mathrm{III}}={ }^{2} \mathrm{Co}^{\mathrm{II}}\right]\right.$ and $\left.\left[{ }^{2} \mathrm{Ru}^{\mathrm{III}}={ }^{4} \mathrm{Co}^{\mathrm{II}}\right]\right)$ are solvated in different ways.

The electronic configurations of the investigated states of $[\mathrm{Ru}=\mathrm{Co}]$ are shown in the bottom of figure 1. The antibonding $\mathrm{e}_{\mathrm{g}}$ orbitals are localized on the Co centre, governing the Co-N bond elongation. In the following, we abbreviate the electronic ground state: $\left[{ }^{1} \mathrm{Ru}{ }^{\mathrm{II}}={ }^{1} \mathrm{Co}^{\mathrm{III}}\right]$ as GS, the low-spin, charge-separated intermediate ${ }^{3}\left[{ }^{2} \mathrm{Ru}^{\mathrm{III}}={ }^{2} \mathrm{Co}^{\mathrm{II}}\right]$, as LS, and finally the high-spin state as HS: $\left[{ }^{2} \mathrm{Ru}^{\mathrm{III}}={ }^{4} \mathrm{Co}^{\mathrm{II}}\right]$.

\section{Computational Methods}

The gas-phase $[\mathrm{Ru}=\mathrm{Co}]$ geometries of the three electronic states were fully relaxed using the quantum chemical code ORCA, ${ }^{42}$ at the $\mathrm{PBE} / \mathrm{TZVP}{ }^{43-45}$ level, and a further relax- 
ation was carried out using the conductor-like screening model ${ }^{46}$ (COSMO). The optimized geometries were confirmed to correspond to minimas on the potential energy surfaces by numerical frequency calculations. These simulations were carried out using the grid-based finite-difference DFT code GPAW, ${ }^{26,27}$ also using the PBE functional (as mandated by computational requirements of the QM/MM MD simulations), using numerical linear combinations of atomic orbitals as the basis in which to represent the pseudo wave functions. ${ }^{47}$ Operations on the electronic density and the potential were still carried out exclusively on the real space grid. Triple- $\zeta$-size basis functions were employed for the metals, double- $\zeta$ for the rest of the atoms, and a grid spacing of $0.18 \AA$. The GS geometry was then placed in a 32x32x44 A box of thermally pre-equilibrated acetonitrile (ACN) molecules, modelled with a classical, rigid, three-point interaction potential. ${ }^{48}$ Non-bonded parameters for the complex were chosen from the UFF force field. ${ }^{49}$ Solvent molecules overlapping the $[\mathrm{Ru}=\mathrm{Co}]$ complex were removed, giving a total of $436 \mathrm{ACN}$ molecules. The dynamics simulations were performed within our own QM/MM MD framework, using the Atomic Simulation Environment $(\mathrm{ASE})^{50}$ to electrostatically embed the QM subsystem in the MM solvent, following an additive explicit coupling scheme. ${ }^{23-25} \mathrm{~A}$ dynamics simulation was started in the GS electronic configuration, and thermalized to $300 \mathrm{~K}$ using a Langevin-type thermostat on the solvent molecules. From the equilibrated part of this trajectory, 10 LS and 13 HS trajectories were started at 1 ps intervals, using unrestricted DFT, and a small finite Fermi-Dirac distribution of the occupation numbers, to ensure stable convergence at every time step. The sampling was carried out by initially setting the magnetic moment on each of the metal centres to match the electronic configurations of each of the excited states, as shown in the bottom of figure 1. The total magnetic moment of the complex was then kept fixed during the MD sampling. For a total triplet multiplicity however, the $\left[{ }^{3} \mathrm{Ru}^{\mathrm{II}}={ }^{1} \mathrm{Co}^{\mathrm{II}}\right]$ state is in principle also viable, and if the energy difference between this state and the one labelled LS is small compared to $k_{B} T$, this state will also be sampled in the MD runs, when the solvent-arrangement and interplay on the complex structure will favour the $\left[{ }^{3} \mathrm{Ru}{ }^{\mathrm{II}}={ }^{1} \mathrm{Co}^{\mathrm{III}}\right]$ state. These two states 
can be distinguished in the simulations using the local magnetic moments of the Co- and Ru-centres, as described in the SI. The trajectories were then re-equilibrated until the solvent temperature and solute bond distances were stable. The following analysis is based on equilibrated 2 fs timestep trajectories amounting to a total of 16 ps for the HS state, and 31 ps for the GS and LS state.

\section{Results \& Discussion}

\section{Energetics and Orbital Studies}

Figure 2 shows the thermally averaged HOMO-LUMO gap obtained by histogramming the difference between HOMO and LUMO orbital energies. The presence of the explicit solvent seems to reduce the HOMO-LUMO gap in both the GS and HS state, compared to conventional geometry optimizations by energy minimization, utilizing continuum solvent models as an approximation for the solvent interaction. ${ }^{9}$

In the LS state, the gap is even more drastically reduced, such that over time, configurations exist where the LUMO is thermally accessible at $300 \mathrm{~K}$, indicating a strong energetic overlap with the bridge-localized LUMO. Indeed, as will be analyzed in depth in figure 3, for the configurations with small gaps, the HOMO is much more bridge-localized.

The lifetime of the LS state in the forward electron transfer cascade is shorter than the thermalization. ${ }^{3}$ The thermally accessible configurations shown for a thermalized molecule at room temperature in Figure 2 thus represents the lower energetic limit of the localization of the LS state on the Co centre during the forward electron transfer; the actual, out-of-equilibrium transfer will have more energy available for driving the process. This coupling explains how the bridge-localized state improves the electron transfer rate, compared to closely related complexes. ${ }^{36,51,52}$ The notion of the bridge-localized state influencing the

charge transfer has been previously hypothesized, ${ }^{3}$ but the experimental limitations have demanded theoretical studies such as the present one to demonstrate the underlying mech- 


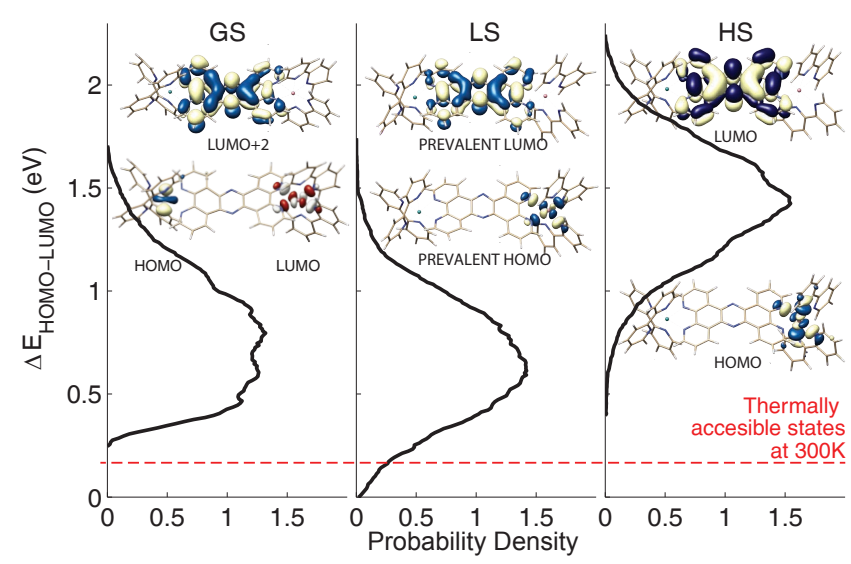

Figure 2: HOMO-LUMO gaps in the three electronic states of $[\mathrm{Ru}=\mathrm{Co}]$. The gaps are produced by histogramming difference in energy between the LUMO and the HOMO orbitals at each frame for all the production simulations carried out. We observe a widening of the gap in the HS state, impeding electronic access to the bridge-localized state. The area under the red dashed line is thermally accessible to the system at $300 \mathrm{~K}$, as achieved by our unrestrained sampling (see figure 4). In accordance with previous static calculations, ${ }^{3}$ a virtual,higher-lying, bridge localized orbital is found in the GS. When the LS HOMO is populated, its character is very dependent on geometric structure and on its solvent surroundings, as discussed in the text and in figure 3. Here, the most prevalent versions are depicted.

anism for the observed phenomenon.

In the HS state, the HOMO-LUMO gap to the bridge-localized state has widened significantly, reducing the energetic coupling and stabilising the charge separated electronic configuration, localizing the charge on the model active-site Co-centre.

In figure 3, one of the LS trajectories was selected for closer scrutiny, correlating visual inspection of the HOMO orbital and the $\mathrm{Co}-\mathrm{N}$ bond distances. Due to thermal fluctuations from the solvent, the available phase space at $k_{B} T$ allows both for configurations where the HOMO orbital has $\mathrm{d}_{\mathrm{x}^{2}-\mathrm{y}^{2}}$ character, and $\mathrm{d}_{\mathrm{z}^{2}}$ character. The plot background is shaded $\mathrm{red} / \mathrm{pink}$ if the antibonding HOMO orbital is localized in the space between the Co-N pairs . If not, it is shaded blue. The red/pink distinction is based on whether the HOMO is $\mathrm{d}_{\mathrm{x}^{2}-\mathrm{y}^{2}}$ or $d_{z^{2}}$. When plotted using the same isosurface value, we also observe intervals where the orbital extends to the bridge section of the molecule, as is the case around 3600 fs on the plot. The intrinsic absence of electronic dynamics in BOMD simulations results in an adia- 


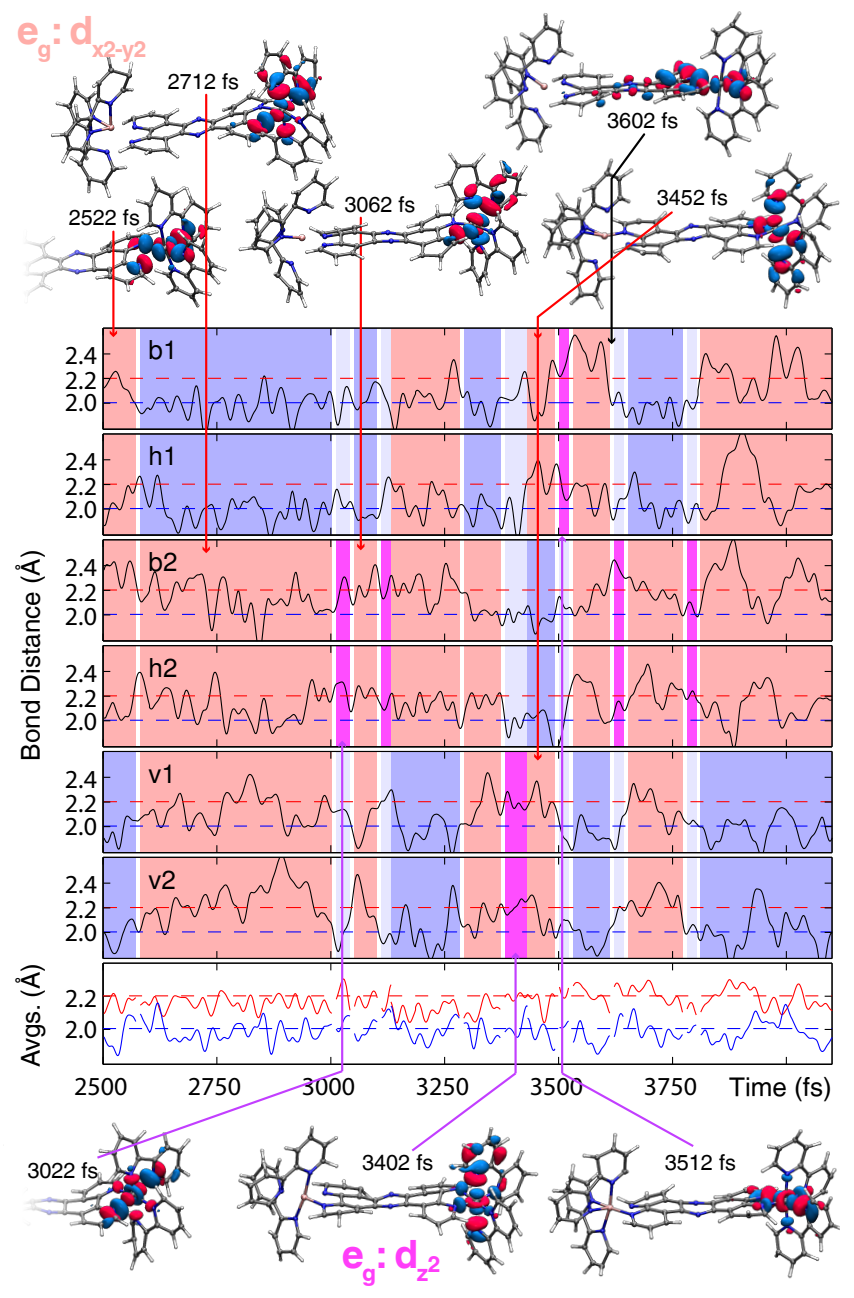

Figure 3: A deeper look into one of the LS trajectories, shedding light upon the charge separated state. LS HOMO orbitals were exported to cube files every 10th fs, and plotted with an isovalue of $0.1 .{ }^{53}$ Whether the HOMO was $\mathrm{d}_{\mathrm{x}^{2}-\mathrm{y}^{2}}$ or $\mathrm{d}_{\mathrm{z}^{2}}$ was confirmed by inspection of the atomic coefficients of molecular orbitals extracted from the converged wave functions. If the HOMO is $\mathrm{d}_{\mathrm{x}^{2}-\mathrm{y}^{2}}$, and localized around a bond, the background of that bond length plot has been shaped red. If the HOMO is $\mathrm{d}_{\mathrm{z}^{2}}$, the shading is pink. If a Co-N pair is not encompassed by the $e_{\mathrm{g}}$ orbital, the trajectory background is shaded blue. The lowest subplot is made from averaging the trajectories with red/pink backgrounds (antibonding) into the red curve, and the blue into the blue curve. These configuration-based averages oscillate around $2.17 \AA$ and $1.99 \AA$, values very similar to the HS and GS averages. At 3602 fs we

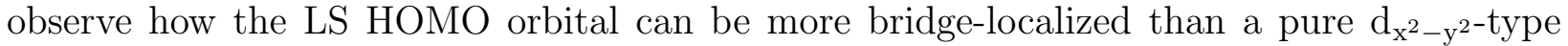
orbital. 
batic representation of the orbital flips and inversions, always sampling the electronic state possessing the lowest energy. The MD is thus solely used as a tool for obtaining thermal distributions. Even so, we hypothesize that the adiabatic trajectories will result in structural distributions which reflect the actual thermal electronic distributions, and seek to substantiate this hypothesis.

Since a single Co-centre e $_{\mathrm{g}}$-orbital is occupied in the LS state, it should be Jahn-Teller (JT) active. Keeping the previously mentioned limitations of the employed model in mind, the simulations can be used to assess an adiabatic description of the dynamic JT behaviour in

solvated systems, as previously done on solvated single-atom ions. ${ }^{54-56}$ If bond distances of the red and blue patches are averaged respectively, we recover distributions with individual bond averages that give the long/short bond length distortion known from the static JT model. ${ }^{57,58}$ However, if all individual bonds are averaged over their entire trajectories, indiscriminately to the orbital arrangement, this distortion vanishes, and we obtain 6 distributions with equal bond lengths, since both $\mathrm{d}_{\mathrm{x}^{2}-\mathrm{y}^{2}}-\mathrm{HOMO}$ and the $\mathrm{d}_{\mathrm{z}^{2}}$-HOMO state are part of the overall accessible phase space of the solute in the solvent at $k_{B} T$. Thus, we underline this subtlety of the mechanism of solvent fluctuations 'smearing out' JT distortions: The JT active state is still distorted when solvated, as we do not find 6 equally long bonds at all sampled MD frames, but the JT distortion is smeared out in the ergodic average.

\section{Thermally Averaged Structures}

The underlying JT behaviour described in figure 3 results in the net distribution shown in figure 4 , bottom. In the HS state, both antibonding $\mathrm{e}_{\mathrm{g}}$ orbitals are occupied, giving rise to the maximum bond elongation. Reiterating from figure 1, only one of the $\mathrm{e}_{\mathrm{g}}$ orbitals is occupied in the LS state. We have seen how occupation of the $\mathrm{d}_{\mathrm{x}^{2}-\mathrm{y}^{2}}$ orbital gives rise to an elongation in 4 out of the 6 bonds, and how filling the $\mathrm{d}_{\mathrm{z}^{2}}$ orbital results in an elongation in 2 out of the 6 bonds. Both of the configurations are available to the LS state at $k_{B} T$ in solution, as explained in figure 3. In order to ascertain which of the two $\mathrm{e}_{\mathrm{g}}$ orbitals posses 
the lowest energy in the thermal average, we perform the analysis shown in figure 4, bottom. Since we have observed how the JT distortion is smeared out in the thermal average, we can use linear combinations of the GS and HS distributions of all 6 bonds to reconstruct the LS distribution for all 6 bonds, $\Gamma_{\mathrm{avg}}^{\mathrm{LS}}(r)$ :

$$
\Gamma_{\mathrm{avg}}^{\mathrm{LS}}(r)=\alpha \Gamma_{\mathrm{avg}}^{\mathrm{GS}}(r)+(1-\alpha) \Gamma_{\mathrm{avg}}^{\mathrm{HS}}(r)
$$

Remembering how the two $\mathrm{e}_{\mathrm{g}}$-orbitals govern the bond elongation, if the only accessible phase-space configuration had $\mathrm{d}_{\mathrm{z}^{2}}$ as $\mathrm{HOMO}$, then the weight $\alpha$ should be equal to $2 / 6$ (plotted as black circles in figure 4, bottom). Conversely, if the HOMO could only be $\mathrm{d}_{\mathrm{x}^{2}-\mathrm{y}^{2}}$, $\alpha=4 / 6$ (red circles). If the analysis of the single trajectory in figure 3 is representative of the total sampled phase space, we would expect $\alpha$ to be slightly less than $4 / 6$, since the HOMO is mostly $\mathrm{d}_{\mathrm{x}^{2}-\mathrm{y}^{2}}$, but not exclusively. This result is precisely what we obtain if we fit $\alpha$, confirming that (1) the cutout of the single trajectory in figure 3 is representative of the entire simulation phase space, and (2) that our hypothesis of being able to produce distributions reflecting the actual thermal, electronic distributions of the system from adiabatic trajectories is possible, in this case.

We note that previous geometry optimization simulations with a different choice of functional and basis set resulted in the elongation of $2 / 6$ bonds, indicating that in the static, gas-phase/implicit solvation picture, the $\mathrm{d}_{\mathrm{z}^{2}}$-orbital has the lower energy of the two $\mathrm{e}_{\mathrm{g}}$ orbitals. ${ }^{9}$ Apart from the the fact that inclusion of explicit solvent and thermal treatment of the system could account for this discrepancy, we also show in the SI that this result is heavily basis-set sensitive. However, the effects on the bond lengths from explicit solvation and thermal averaging exceed the basis set/functional-dependent discrepancies for the explored basis sets and functionals (see the S.I. for more info).

In order to further connect the thermal averages of the QM/MM MD simulations with the static geometry optimization picture, the Potential of Mean Force (PMF), shown in figure 


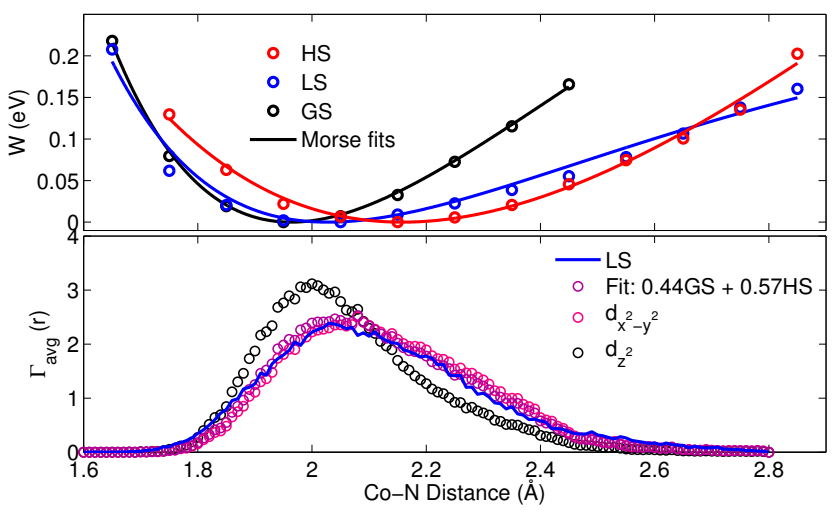

Figure 4: Top: The Co-N Potential of Mean Force (PMF) for each electronic configuration, calculated from the RDFs from the simulations. The fully drawn lines are Morse-potential fits of each PMF. The unbiased sampling show that states are thermally accessible up to over $0.2 \mathrm{eV}$. Bottom: Reconstructing the average Co-N LS distribution from linear combinations of the average GS and HS distributions. $\mathrm{d}_{\mathrm{x}^{2}-\mathrm{y}^{2}}: 1 / 3 \mathrm{GS}+2 / 3 \mathrm{HS} . \mathrm{d}_{\mathrm{z}^{2}}: 2 / 3 \mathrm{GS}+1 / 3 \mathrm{HS}$.

4, top, has been calculated from the pairwise Co-N RDFs, $g_{\mathrm{Co}-\mathrm{N}}(r)$. The full lines represent Morse-potential fits of the form

$$
V(r)=D_{d}\left(1-e^{-a\left(r-r_{0}\right)}\right)^{2}
$$

where $D_{d}$ is the dissociation energy, $r_{0}$ is the equilibrium distance, and $a=\sqrt{k_{0} /\left(2 D_{d}\right)}$ defines the force constant $k_{0}$ of the potential. The GS and HS potentials are well described with this model, yielding $r^{2}$-values of 0.998 and 0.990 , respectively. The LS intermediate has $r^{2}=0.971$ and a systematic deviation from the model is observed at the longer bond distances, further indicating that the LS distribution is, in fact, not a single distribution, but a combination of distributions arising from the dynamically varying characteristics of the HOMO orbital as depicted in figure 3.

The Morse-fitted minima is in good agreement with the geometry relaxations of the systems at $1.963 \AA$ for the GS, $2.058 \AA$ for the LS, and $2.158 \AA$ for the HS state. The geometry optimization carried out in ORCA with the same functional and similarly sized basis set gave average Co-N values of $1.957 \AA, 2.043 \AA$, and $2.129 \AA$ respectively (see the SI for details). The maximum deviation is $1.4 \%$, for the HS state. We note that the thermally averaged 
geometry of the complex, closer to what is usually measured experimentally, will be different from minimum-energy optimized structures, due to the not uncommon anharmonicities of the underlying potentials.

When the thermal averages of each individual Co-N bond is histogrammed, we obtain the results shown in figure 5. For the HS state, the QM/MM MD results successfully reproduce the full experimental $0.20 \AA$ elongation, ${ }^{3}$ when taking the total average of the 6 thermally averaged bonds. When filtering the multimode-distribution in the LS state to only contain the samples from charge-separated intermediate, the bonds elongate on average altogether by $0.12 \AA$.

The figure also shows a visual overview of the thermal averages on the structural changes induced by the excited state electronic configurations. Here, the GS average structure, visualized in the $50 \%$ thermal ellipsoid-representation, is superimposed on the same representation of the LS (top) and HS (bottom) thermal average structure, after removing overall translational (center of mass) and rotational motion, using the Root Mean Square Displacement (RMSD) align tool in the VMD program. ${ }^{53}$ In both excited states, the previously noted broadening of bond length distributions are reflected in the enlarged thermal ellipsoids of the Co-adjacent nitrogen atoms and their neighbouring carbons.

The distortions undertaken by the Co-N bonds drives further structural changes, the thermal averages of which are presented in figure 6 . The figure shows the bite- $(\beta)$ and butterfly- $(\theta)$ angle distributions. The HS $\beta$ decreases by $\sim 6^{\circ}$, consistent with previous studies of similar transition metal complexes. ${ }^{16}$ Congruous with the LS Co-N bond lengths, the LS $\beta$ distributions appear as intermediates between the GS and HS states, with an average decrease of $\sim 3^{\circ}$. For the Co-centre, both angle distributions again widen in the electronically excited states. The $\beta$-angle of both the Co and the Ru centre increases in the excited states, with the $\Delta \beta$-values presented in the figure. 

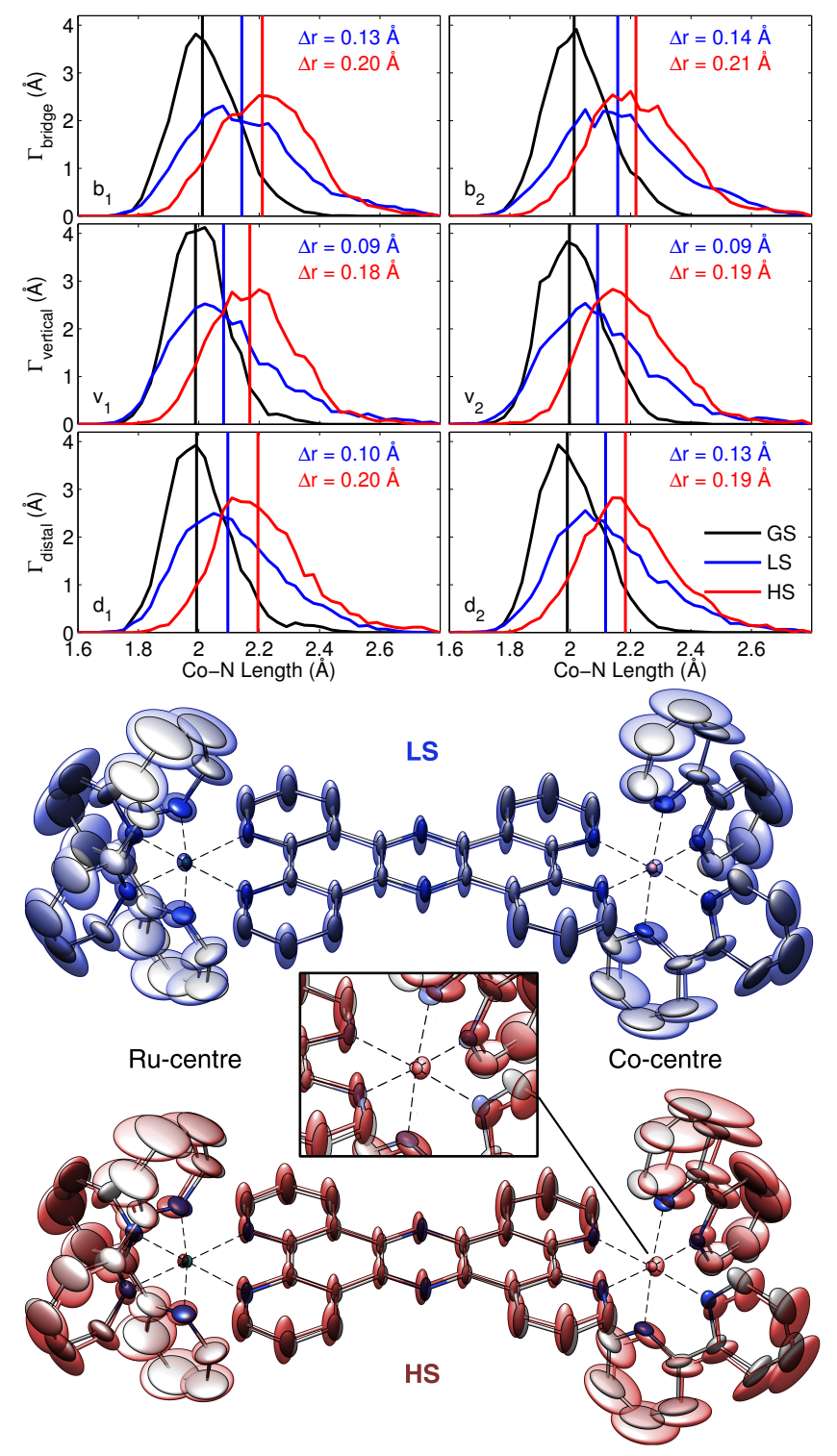

Figure 5: Top: Thermal distributions of Co-N bonds. The black, blue, and red lines represent the average of the thermal distributions of the GS, LS and HS state, respectively. In the LS state, only the MD frames containing the charge-separated electronic configurations are included in the distribution. Bottom: Average structures with thermal ellipsoids of the $[\mathrm{Ru}=\mathrm{Co}]$ complex, showing the $50 \%$ probability isosurfaces of the atomic positions. The hydrogens, constrained in the simulations, are omitted from the visualization. The GS average structure is overlaid both the LS (top) and the HS (bottom) structure, and the cutout focuses on the Co-N expansion in the HS state. 

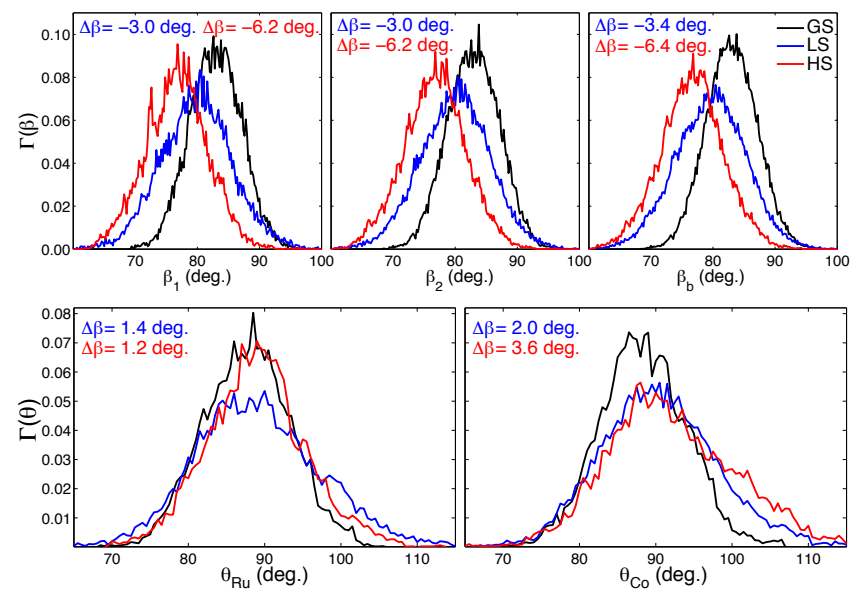

Figure 6: Top: Thermal distributions of Co-centre bite angles $\beta$. Bottom: Thermal distributions of butterfly angles from both metal centres.
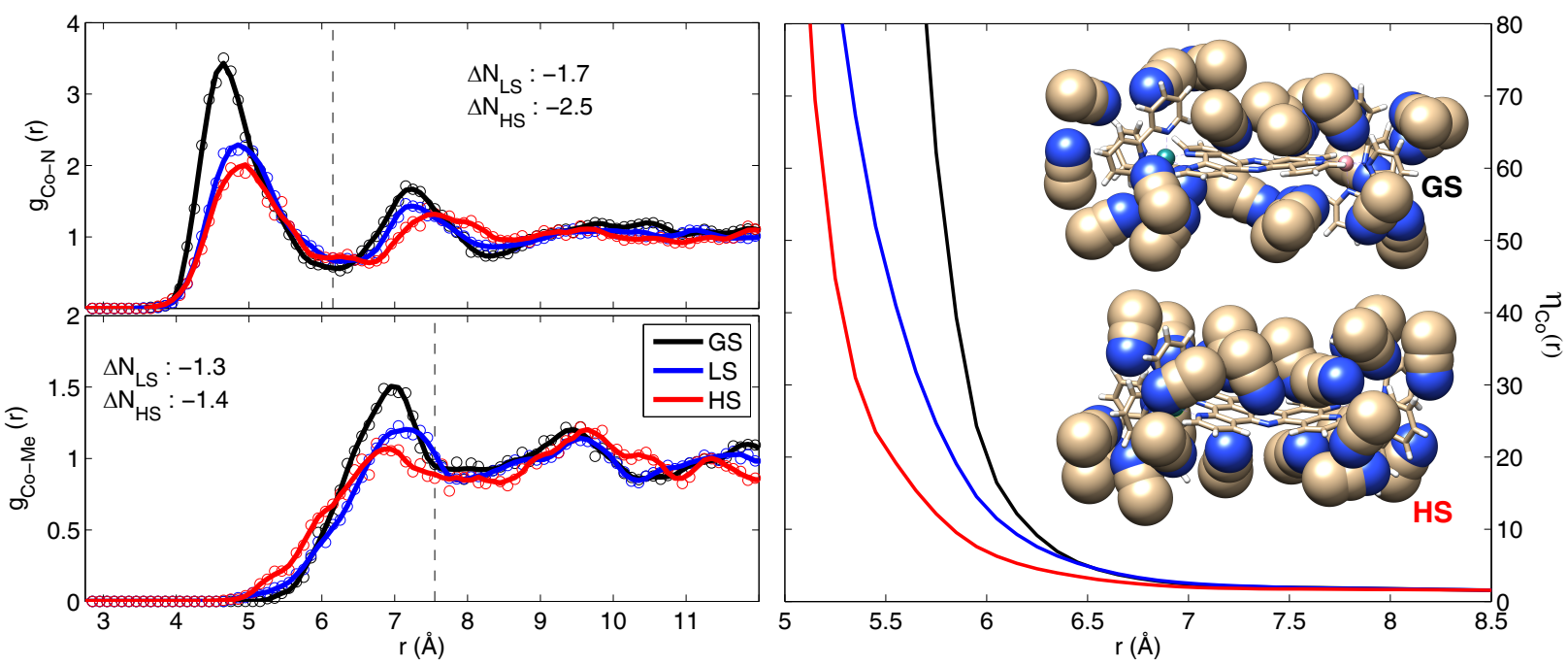

Figure 7: Left plots: The pairwise RDFs between the Co atom and the ACN-N (top) and ACN-Methyl group (bottom) respectively. The fully drawn lines represent a 5th order Savitzky-Golay filter applied on the data. Right: The N:CH3 ratio of the cumulative coordination numbers for Co. The two inset images are snapshots of the GS (top) and HS (bottom) solvent shells, defined by drawing only the full solvent molecules which are within $8 \AA$ of either Ru (left on the images) or Co. 


\section{Solvent Shell Response}

To quantify the solvation shell changes around the Co-centre, we calculated the pairwise radial distribution functions (RDFs), and their associated running coordination numbers, $c n(r)$, using numerical, radial histogramming with a bin size of $0.1 \AA$. The two left plots in figure 7 show the RDFs between Co and the ACN-N (top) and ACN-Methyl group (bottom) respectively. We define the first Co- $\mathrm{N}$ solvation shell distance $R$ to terminate at the dashed line in the top plot, at $R=6.2 \AA$, giving an average of $\sim 10.5$ solvent molecules in the first Cocentre shell in the GS. This number is then used to find the $r$ at which the Co-Me RDF contain the same amount of molecules, making it possible to distinguish between translational and rotational changes in the solvent molecule configuration. If solvent molecules are exclusively moving radially away from (or closer to) the Co-centre due to the excitation (translation), the change in coordination numbers,

$$
\Delta N_{\mathrm{ES}}=c n_{\mathrm{GS}}(R)-c n_{\mathrm{ES}}(R), \mathrm{ES}=\mathrm{LS}, \mathrm{HS}
$$

should be equal for the RDF pairs of each end of the solvent molecule. First, we observe how the extra electron on the Co-centre of the charge-separated states electrostatically reduces the coordination of the electronegative ACN-N ends, as expected. As with similar systems, ${ }^{16}$ we note an expulsion of 1 or more solvent molecules in the excited states, due to a combination of the excitation-induced changes in both electronic and nuclear structures. Since the change of coordination numbers in the excited state is $\Delta N_{\mathrm{Co}-\mathrm{N}}>\Delta N_{\mathrm{Co}-\mathrm{Me}}$, on average, two or three ACN-N ends rotate away from the Co-centre, in addition to the translationally expelled solvent molecule. To further examine the rotational solvation dynamics, figure 7 (right plot) also shows the ratios of the metal- to ACN-Nitrogen and metal to ACN-methyl group running coordination numbers:

$$
\eta_{\mathrm{Co}}(r)=c n_{\mathrm{Co}-\mathrm{N}}(r) / c n_{\mathrm{Co}-\mathrm{Me}}(r)
$$


This ratio illustrates the on-average preferred orientation of the solvent molecules, relative to the Co-centre. At closer ranges, the ratio increases, indicating an overall preferred orientation of pointing the partially negative charged ACN N-end towards the metal, consistent with the overall $5+$ charge on the complex. The Co-ratio decreases upon excitation, indicating that, on average, the N-ends become less oriented towards Co compared to the ground state, while still maintaining some of the preferred N-Co orientation. This is consistent with the Co-centre being reduced in the excited states, which again makes the solvent respond accordingly. Thus, the interactions between solute and solvent atoms help stabilize and localize the separated charge.

\section{Conclusions}

Within this work, we have employed direct dynamics simulations to map the interplay between electronic, structural and solvation dynamics. The simulations have allowed us to explore thermally available structural and solvation configurations for a given electronic state, and we have shown that this information can be used to reconstruct the potentials that are relevant/explored by each electronic state. Where it is possible to compare simulation to data retrieved with state-of-the-art experimental techniques, they are in good agreement. This inspires confidence in our approach, and the calculated results thus both allow us to investigate the nature of states that are too short-lived for thorough experimental investigation, and perhaps more importantly allow us to extrapolate the method to unexplored/not-yet-synthesized molecules. For the system modelled in this work, we have shown how the energetics of the intermediate state help improve the electron transfer rate, a mechanism with advantages that could be taken into account when designing future generations of photocatalytic systems. Our method thus expands on the knowledge accessible from conducted experiments, and also provide the potential for predicting the behavior of currently molecular systems yet to be synthesized. 
The direct dynamics model was shown to provide a very good description of the structure of the spin-relaxed charge-separated state, with the direct dynamics simulations successfully reproducing the observed HS $0.20 \AA \mathrm{Co}-\mathrm{N}$ bond elongation. For the non-spin-relaxed charge-separated state, we observed strong JT-dynamics which static DFT methods struggle to describe, and we showed how it was possible to recover the geometric JT-distortion by correlating geometries with orbitals over trajectories.

Overall, this work represents an exhaustive utilization of the employed model, within its intrinsic limits. Future studies should strive to include more states, coupling of electronic and nuclear dynamics, and the resulting out-of-equilibrium dynamics. However, the current computational frameworks for these models still cannot produce enough data on systems of this size, for a statistically sound comparison to experimental observations of phenomena on the 1-50 ps time scale. The 1-50 ps time scale is inherently important in solvent-mediated processes, which means that direct dynamics simulations of actual dynamics are either limited

to fractions of picoseconds, ${ }^{19,59}$ or needs to employ further approximations to the electronic structure method, like the coarse grained DFT approach. ${ }^{60}$

\section{Associated Content}

\section{Acknowledgement}

The authors thank E. Ö. Jónsson and M. I. Pápai for discussion and feedback.

This work was funded by The Danish Council for Independent Research. S.E. Canton acknowledges funding from SFB 1073.

\section{Supporting Information Available}

Supporting Information Available: Further computational details, preliminary simulations, geometry relaxation results, thermal distributions of torsions. This material is available 
free of charge via the Internet at http://pubs.acs.org/. 


\section{References}

(1) Haldrup, K. et al. Observing Solvation Dynamics with Simultaneous Femtosecond X-ray Emission Spectroscopy and X-ray Scattering. J. Phys. Chem.. B 2016, 120, 1158-1168.

(2) Chergui, M. Ultrafast Photophysics of Transition Metal Complexes. Acc. Chem. Res. 2015, 48, 801-808.

(3) Canton, S. E. et al. Visualizing the non-equilibrium dynamics of photoinduced intramolecular electron transfer with femtosecond X-ray pulses. Nat. Commun. 2015, 6, 6359.

(4) Kim, K. H. et al. Direct observation of bond formation in solution with femtosecond X-ray scattering. Nature 2015, 518, 385-389.

(5) Zhang, W. et al. Tracking Excited-State Charge and Spin Dynamics in Iron Coordination Complexes. Nature 2014, 509, 345.

(6) Kim, K. H.; Kim, J.; Lee, J. H.; Ihee, H. Topical Review: Molecular reaction and solvation visualized by time-resolved X-ray solution scattering: Structure, dynamics, and their solvent dependence. Struct. Dyn. 2014, 1, 011301.

(7) Lemke, H. T. et al. Femtosecond X-ray Absorption Spectroscopy at a Hard X-ray Free Electron Laser: Application to Spin Crossover Dynamics. J. Phys. Chem. A 2013, 117, $735-740$.

(8) Haldrup, M. et al. Guest-Host Interactions Investigated by Time-Resolved X-ray Spectroscopies and Scattering at MHz Rates: Solvation Dynamics and Photoinduced Spin Transition in Aqueous Fe(bipy) ${ }_{3}^{2+}$. J. Phys. Chem. A 2012, 116, 9878-9887.

(9) Kuhar, K.; Fredin, A.; Persson, P. Exploring Photoinduced Excited State Evolution in Heterobimetallic Ru(II)-Co(III) Complexes. J. Phys. Chem. B 2015, 119, 7378-7392. 
(10) Kong, Q.; Kim, J.; Lorenc, M.; Kim, T. K.; Ihee, H.; Wulff, M. Photodissociation Reaction of 1,2-Diiodoethane in Solution: A Theoretical and X-ray Diffraction Study. J. Phys. Chem. A 2005, 109, 10451-10458.

(11) Kong, Q.; Kjær, K. S.; Haldrup, K.; Sauer, S. P. A.; van Driel, T. B.; Christensen, M.; Nielsen, M. M.; Wulf, M. Theoretical study of the triplet excited state of PtPOP and the exciplexes M-PtPOP $(\mathrm{M}=\mathrm{Tl}, \mathrm{Ag})$ in solution and comparison with ultrafast X-ray scattering results. Chem. Phys. 2012, 17, 117-122.

(12) Hunter, B. M.; Villahermosa, R. M.; Exstrom, C. L.; Hill, M. G.; Mann, K. R.; Gray, H. B. M-M bond-stretching energy landscapes for $\mathrm{M}_{2}(\text { dimen })_{4}^{2+}(\mathrm{M}=\mathrm{Rh}$, Ir; dimen =1,8-diisocyanomenthane) complexes. Inorg. Chem. 2012, 51, 6898.

(13) Pápai, M.; Vankó, G. On Predicting Mssbauer Parameters of Iron-Containing Molecules with Density-Functional Theory. J. Chem. Theory Comput. 2013, 9, 5004-5020.

(14) Dixon, I. M.; Alary, F.; Boggio-Pasqua, M.; Heully, J.-L. Reversing the relative 3MLCT3MC order in Fe(ii) complexes using cyclometallating ligands: a computational study aiming at luminescent Fe(ii) complexes. Dalton Trans. 2015, 44, 13498-13503.

(15) Vankó, G. et al. Detailed Characterization of a Nanosecond-Lived Excited State: X-ray and Theoretical Investigation of the Quintet State in Photoexcited $\left[\mathrm{Fe}(\text { terpy })_{2}\right]^{2+} . J$. Phys. Chem. C 2015, 119, 5888-5902.

(16) Daku, L. M. L.; Hauser, A. Ab Initio Molecular Dynamics Study of an Aqueous Solution of $\left[\mathrm{Fe}(\mathrm{bpy})_{3}\right](\mathrm{Cl})_{2}$ in the Low-Spin and in the High-Spin States. J. Phys. Chem. Letters 2010, 1, 1830-1835.

(17) Pham, V.; Tavernelli, I.; Milne, C.; van der Veen, R.; DAngelo, P.; Bressler, C.; Chergui, M. The solvent shell structure of aqueous iodide: X-ray absorption spectroscopy and classical, hybrid QM/MM and full quantum molecular dynamics simulations. Chem. Phys. 2010, 371, $24-29$. 
(18) Pham, V.-T.; Penfold, T. J.; van der Veen, R. M.; Lima, F.; Nahhas, A. E.; Johnson, S. L.; Beaud, P.; Abela, R.; Bressler, C.; Tavernelli, I.; Milne, C. J.; Chergui, M. Probing the Transition from Hydrophilic to Hydrophobic Solvation with Atomic Scale Resolution. J. Am. Chem. Soc. 2011, 133, 12740-12748.

(19) Tavernelli, I.; Curchod, B. F. E.; Rothlisberger, U. Nonadiabatic molecular dynamics with solvent effects: A LR-TDDFT QM/MM study of ruthenium (II) tris (bipyridine) in water. Chem. Phys. 2011, 391, 101-109.

(20) Penfold, T. J.; Curchod, B. F. E.; Tavernelli, I.; Abela, R.; Rothlisberger, U.; Chergui, M. Simulations of X-ray absorption spectra: the effect of the solvent. Phys. Chem. Chem. Phys. 2012, 14, 9444-9450.

(21) Penfold, T.; Tavernelli, I.; Doemer, M.; Abela, R.; Rthlisberger, U.; Chergui, M. Solvent rearrangements during the transition from hydrophilic to hydrophobic solvation. Chem. Phys. 2013, 410, $25-30$.

(22) Tavernelli, I. Nonadiabatic Molecular Dynamics Simulations: Synergies between Theory and Experiments. Acc. Chem. Res. 2015, 48, 792-800.

(23) Dohn, A. O.; Henriksen, N. E.; Møller, K. B. Transient Changes in Molecular Geometries and How to Model Them; Springer International Publishing, 2014.

(24) Jónsson, E. Ö.; Thygesen, K. S.; Ulstrup, J.; Jacobsen, K. W. Computational Approach to Electron Charge Transfer Reactions; 2014.

(25) Dohn, A. O.; Jónsson, E. O.; Kjær, K. S.; B. van Driel, T.; Nielsen, M. M.; Jacobsen, K. W.; Henriksen, N. E.; Møller, K. B. Direct Dynamics Studies of a Binuclear Metal Complex in Solution: The Interplay Between Vibrational Relaxation, Coherence, and Solvent Effects. J. Phys. Chem.. Lett. 2014, 5, 2414-2418. 
(26) Mortensen, J.; Hansen, L.; Jacobsen, K. W. Real-space grid implementation of the projector augmented wave method). Phys. Rev. B 2005, 71, 035109.

(27) Enkovaara, J. et al. Electronic structure calculations with GPAW: a real-space implementation of the projector augmented-wave method. Journal of Physics: Condensed matter 2010, 22, 253202.

(28) Blöchl, P. E. Projector Augmented-Wave Method. Phys. Rev. B 1994, 50, 17953.

(29) Barthel, E. R.; Martini, I. B.; Schwartz, B. J. How Does the Solvent Control Electron Transfer? Experimental and Theoretical Studies of the Simplest Charge Transfer Reaction. J. Phys. Chem. B 2001, 105, 12230-12241.

(30) Kao, Y.-T.; Guo, X.; Yang, Y.; Liu, Z.; Hassanali, A.; Song, Q.-H.; Wang, L.; Zhong, D. Ultrafast Dynamics of Nonequilibrium Electron Transfer in Photoinduced Redox Cycle: Solvent Mediation and Conformation Flexibility. J. Phys. Chem. B 2012, 116, 91309140.

(31) Rau, S.; Schfer, B.; Gleich, D.; Anders, E.; Rudolph, M.; Friedrich, M.; Grls, H.; Henry, W.; Vos, J. G. A Supramolecular Photocatalyst for the Production of Hydrogen and the Selective Hydrogenation of Tolane. Angew. Chem. Int. Ed. 2006, 45, 62156218.

(32) Ozawa, H.; Haga, M. A.; Sakai, K. A photo-hydrogen-evolving molecular device driving visible-light-induced EDTA-reduction of water into molecular hydrogen. J. Am. Chem. Soc. 2006, 128, 4926-4927.

(33) Chiorboli, C.; Bignozzi, C.; Scandola, F. Photophysics of Dinuclear Ru (II) and Os (II) Complexes Based on the Tetrapyrido phenazine (tpphz) Bridging Ligand. Inorg. Chem. 1999, 38, 2402-2410. 
(34) Rau, S.; Walther, D.; Vos, J. G. Inspired by nature: light driven organometallic catalysis by heterooligonuclear $\mathrm{Ru}(\mathrm{II})$ complexes. Dalton Trans. 2007, 915-919.

(35) Tschierlei, S.; Presselt, M.; Kuhnt, C.; Yartsev, A.; Pascher, T.; Sundström, V.; Karnahl, M.; Schwalbe, M.; Schäfer, B.; Rau, S.; Schmitt, M.; Dietzek, B.; Popp, J. Photophysics of an intramolecular hydrogen-evolving Ru-Pd photocatalyst. Chem. - Eur. J. 2009, 15, 7678-7688.

(36) Song, X.; Lei, Y.; Wallendal, S. V.; Perkovic, M. W.; Jackman, D. C.; Endicott, J. F.; Rillema, D. P. Photoinduced electron-transfer processes involving covalently linked ruthenium and cobalt polypyridyl complexes: comparison of electronic coupling in bridged and nonbridged ruthenium and cobalt complexes. J. Phys. Chem. 1993, 97, $3225-3236$.

(37) Torieda, H.; Nozaki, K.; Yoshimura, A.; Ohno, T. Temperature-Independent Rate of Electron-Transfer between a Cobalt(II) and a Ruthenium(III) of Doublet Electronic Configuration. J. Phys. Chem. A 2002, 106, 11034-11044.

(38) Torieda, H.; Nozaki, K.; Yoshimura, A.; Ohno, T. Low Quantum Yields of Relaxed Electron Transfer Products of Moderately Coupled Ruthenium(II)-Cobalt(III) Compounds on the Subpicosecond Laser Excitation. J. Phys. Chem. A 2004, 108, 4819-4829.

(39) Yoshimura, A.; Torieda, H.; Ohno, T. Doublet-Quartet Intersystem Crossing and Electron Transfer of Cobalt ( II ) Moieties in. J. Phys. Chem 2004, 108, 2149-2154.

(40) Karnahl, M.; Tschierlei, S.; Kuhnt, C.; Dietzek, B.; Schmitt, M.; Popp, J.; Schwalbe, M.; Krieck, S.; Gorls, H.; Heinemann, F. W.; Rau, S. Synthesis and characterization of regioselective substituted tetrapyridophenazine ligands and their $\mathrm{Ru}(\mathrm{ii})$ complexes. Dalton Trans. 2010, 39, 2359-2370.

(41) Canton, S. E. et al. Toward Highlighting the Ultrafast Electron Transfer Dynamics at the Optically Dark Sites of Photocatalysts. J. Phys. Chem. Lett. 2013, 4, 1972-1976. 
(42) Neese, F. ORCA - An ab initio, DFT and semiempirical SCF-MO package - version 2.8. 2010 .

(43) Perdew, J. P.; Burke, K.; Ernzerhof, M. Generalized gradient approximation made simple. Phys. Rev. Lett. 1996, 77, 3865-3868.

(44) Weigend, F.; Ahlrichs, R. Balanced basis sets of split valence, triple zeta valence and quadruple zeta valence quality for H to Rn: Design and assessment of accuracy. Phys. Chem. Chem. Phys. 2005, 7, 3297-3305.

(45) Schäfer, A.; Huber, C.; Ahlrichs, R. Fully optimized contracted Gaussian basis sets of triple zeta valence quality for atoms Li to Kr. J. Chem. Phys. 1994, 100, 5829-5835.

(46) Sinnecker, S.; Rajendran, A.; Klamt, A.; Diedenhofen, M.; Neese, F. Calculation of Solvent Shifts on Electronic G-Tensors with the Conductor-Like Screening Model (COSMO) and its Self-Consistent Generalization to Real Solvents (COSMO-RS). J. Phys. Chem. A 2006, 110, 2235-2245.

(47) Larsen, A. H.; Vanin, M.; Mortensen, J. J.; Thygesen, K. S.; Jacobsen, K. W. Localized Atomic Basis Set in the Projector Augmented Wave Method. Phys. Rev. B 2009, 80, 195112.

(48) Guardia, E.; Pinzón, R.; Casulleras, J.; Orozco, M.; Luque, F. J. Comparison of different three.site interaction potentials for liquid acetonitrile. Mol. Simul. 2001, 26, 287.

(49) Rappe, A.; Casewit, C.; Colwell, K.; III, W. G.; Skiff, W. UFF, a Full Periodic Table Force Field for Molecular Mechanics and Molecular Dynamics Simulations. J. Am. Chem. Soc. 1992, 114, 10024-10035.

(50) Bahn, S. R.; Jacobsen, K. W. An object-oriented scripting interface to a legacy electronic structure code. Comput. Sci. Eng. 2002, 4, 55. 
(51) Chiorboli, C.; Rodgers, M. A.; Scandola, F. Ultrafast processes in bimetallic dyads with extended aromatic bridges. Energy and electron transfer pathways in tetrapyridophenazine-bridged complexes. J. Am. Chem. Soc. 2013, 125, 483-491.

(52) Canton, S. E. et al. Watching the dynamics of electrons and atoms at work in solar energy conversion. Faraday Discuss. 2015, 185, 51-68.

(53) Humphrey, W.; Dalke, A.; Schulten, K. VMD: Visual Molecular Dynamics. J. Mol. Graphics 1996, 14, 33-38.

(54) Schwenk, C. F.; Rode, B. M. Extended ab initio quantum mechanical/molecular mechanical molecular dynamics simulations of hydrated $\mathrm{Cu}^{2+}$. J. Chem. Phys. 2003, 119, 9523-31.

(55) Blumberger, J.; Bernasconi, L.; Tavernelli, I.; Vuilleumier, R.; Sprik, M. Electronic Structure and Solvation of Copper and Silver Ions: A Theoretical Picture of a Model Aqueous Redox Reaction. J. Am. Chem. Soc. 2004, 126, 3928-3938.

(56) Kritayakornupong, C. The Jahn-Teller Effect of the $\mathrm{Cr}^{2+}$ Ion in Aqueous Solution: $\mathrm{Ab}$ Initio QM/MM Molecular Dynamics Simulations. J. Comput. Chem. 2007, 29, 115121.

(57) Jahn, H. A.; Teller, E. Stability of Polyatomic Molecules in Degenerate Electronic States. I. Orbital Degeneracy. Proc. R. Soc. London, Ser. A 1937, 161, 220-235.

(58) Bersuker, I. The Jahn-Teller Effect; Cambridge University Press, 2006.

(59) Jailaubekov, A. E.; Willard, A. P.; Tritsch, J. R.; Chan, W.-L.; Sai, N.; Gearba, R.; Kaake, L. G.; Williams, K. J.; Leung, K.; Rossky, P. J.; Zhu, X.-Y. Hot charge-transfer excitons set the time limit for charge separation at donor/acceptor interfaces in organic photovoltaics. Nat. Mater. 2013, 12, 66-73. 
(60) Woiczikowski, P. B.; Steinbrecher, T.; Kubar, T.; Elstner, M. Nonadiabatic QM/MM Simulations of Fast Charge Transfer in Escherichia coli DNA Photolyase. J. Phys. Chem. B 2011, 115, 9846-9863. 


\section{For Table of Contents Only}

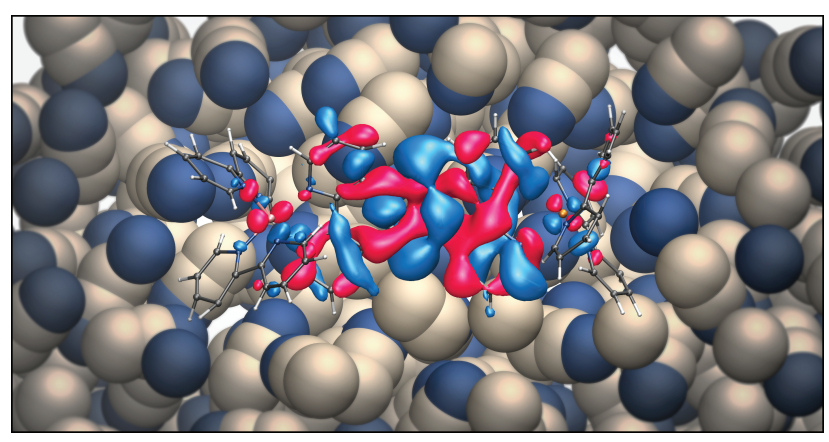

The mechanism for electron transfer in a hetero-dinuclear photocatalysis complex depends on an intermediate charge separated state, stabilized by the solvent. Jahn-Teller distortion effects are averaged out in the thermal average, but can be recovered in individual molecular geometries. 